\title{
Velocidad de ajuste del precio de las acciones por sector económico en la Bolsa Mexicana de Valores
}

Verónica Patricia

Rodríguez Vázquez

Profesora investigadora.

Departamento de Finanzas y

Contaduría. Universidad de las

Américas, Puebla

veronicap.rodriguez@udlap.mx

Maribel Ávila

Espinosa

Asesora financiera y contable

de PYMES

maribel_avila85@yahoo.com

Alejandra Torres

González

Analista de costos

ale_ene10@yahoo.com

\section{Resumen}

Este estudio analiza la velocidad de ajuste del precio de las acciones de la Bolsa Mexicana de Valores de enero de 1998 a diciembre de 2007; para ello, se agruparon las acciones por sectores económicos y se compararon con el Índice de Precios y Cotizaciones (IPC). Asimismo, se clasificó a los sectores económicos en fuertes y débiles, dependiendo del porcentaje de influencia dentro de la muestra del IPC. Entre los sectores fuertes se ubicó a comunicaciones y transportes, comercio, construcción e industria extractiva; mientras que en los débiles a transformación, servicios y varios. El modelo de regresión utilizado fue el propuesto por Marshall y Walker (2002), con el que se buscó comprobar que los sectores fuertes ajustaban más rápidamente sus precios que los sectores débiles con respecto a la rentabilidad del mercado. Se encontró que existe evidencia de que los retornos de los sectores fuertes se adelantan a los retornos de los sectores débiles, principalmente porque los primeros ajustan más rápidamente sus precios a los movimientos del mercado.

Palabras clave: velocidad de ajuste, rentabilidad, IPC, índices sectoriales, Bolsa Mexicana de Valores.

No. 228, mayo-agosto 2009: 67-81 
Verónica Patricia Rodríguez Vázquez, Maribel Ávila Espinosa y Alejandra Torres González

\title{
Speed of price adjustment by economic sector in the mexican stock market
}

\begin{abstract}
Building on the work of Marshall and Walker (2002) this paper analyzes the speed of price adjustment to size and trade volume in the mexican stock markets from January 1998 to December 2007. Shares are grouped by economic sectors and then compared with the index of equity returns (Índice de Precios y Cotizaciones, IPC). Economic sectors are grouped into strong and weak ones, depending upon their influence on the IPC index. Among the strong sectors we find communications and transportation, commerce, construction and extractive industries. On the other hand, among the weak sectors we find transformation industry, services and miscellaneous. Results show that strong sectors adjust their prices faster than the weak ones with respect to market returns.
\end{abstract}

Keywords: mexican stock markets, price adjustment, IPC, index of equity returns.

\section{Introducción}

A lo largo de los años, académicos e inversionistas se han interesado en el papel que juega el volumen de transacciones en la predicción de retornos futuros. Sin embargo, desde principios de la década de los años sesenta del siglo pasado han existido diferentes teorías que afirman que no se pueden obtener ganancias extraordinarias, utilizando información adicional a la histórica, por estar ya reflejada en el precio de las acciones. A esta teoría se le conoce como "hipótesis de la eficiencia de los mercados". Por otra parte, en estudios realizados por Brennan, Jegadeesh y Swaminathan (1993), así como por Chorida y Swaminathan (2000), se ha encontrado que los retornos de las empresas con altos volúmenes de transacciones anticipan los retornos del resto, con lo que los inversionistas pueden obtener beneficios adicionales.

Por lo anterior, surge la necesidad de detectar, a través del poder predictivo del ajuste del precio de las acciones, el momento en el cual se pueden comprar acciones a un precio bajo para vender posteriormente a un precio alto. Esto permitirá a los inversionistas y analistas financieros contar con información adicional que les ayude en la toma de decisiones relacionada con la formación de portafolios de inversión y a la compra y venta de acciones con el fin de lograr mejores rendimientos. 
En este estudio se analiza la predictibilidad del ajuste del precio de las acciones de la Bolsa Mexicana de Valores, por sectores económicos, con base en retornos semanales. Los sectores se clasifican en fuertes y débiles de acuerdo con el peso que tiene cada uno de éstos en la muestra del Índice de Precios y Cotizaciones (IPC); en los primeros se encuentran: comunicaciones y transportes, comercio, construcción e industria extractiva; en lo segundos: transformación, servicios y varios. Por otra parte, los retornos semanales serán los valores para cada sector y para el IPC de miércoles a miércoles, con el fin de minimizar el efecto día de la semana. El periodo analizado comprende de enero de 1998 a diciembre de 2007.

El objetivo de nuestra investigación es comprobar que los sectores fuertes ajustan más rápidamente sus precios con respecto a la rentabilidad del mercado, que los sectores débiles. Para llevar a cabo lo anterior, se utilizó la regresión Dimson (1979), sugerida por Marshall y Walker (2002), quienes estudiaron distintas características de las distribuciones de frecuencia de los retornos accionarios semanales en la bolsa de Santiago de Chile de 1990 a 2000. Aunado a ello analizaron la velocidad de ajuste relacionada con información del mercado y la velocidad de ajuste entre portafolios utilizando vectores autorregresivos. En el caso de la velocidad de ajuste con respecto al mercado encontraron evidencia de que el mercado demora en incorporar información a los precios de las acciones de empresas pequeñas y sin liquidez.

De esta forma, surge la idea de buscar evidencia de este último efecto dentro de la Bolsa Mexicana de Valores y, al mismo tiempo, observar el comportamiento que siguen los sectores económicos con respecto al IPC, por lo que para este estudio se toma como referencia la regresión Dimson del estudio de Marshall y Walker (2002), que es la que atañe a la velocidad de ajuste relacionada con información del mercado.

\section{Revisión de la bibliografía}

Para la realización de este estudio es necesario conocer el funcionamiento y las características de la Bolsa Mexicana de Valores (BMv), que es una organización privada que brinda las facilidades necesarias para que sus miembros realicen negociaciones de compra-venta de valores, tales como acciones de sociedades o compañías anónimas, bonos públicos y privados, certificados, títulos de participación y una variedad de instrumentos de inversión. En general, las bolsas de valores fomentan el ahorro y la inversión a largo plazo, fortaleciendo con ello al mercado 
de capitales e impulsando el desarrollo económico y social de los países donde funcionan. Asimismo, en estas bolsas existen índices bursátiles, que son un instrumento de los mercados bursátiles para seguir la evolución del conjunto de las acciones cotizadas. La mayor parte de los índices se constituyen con una selección de acciones que pretenden representar a la totalidad. Los índices bursátiles son una referencia cada vez más importante para los gestores de cartera, así como lo son también en la oferta de nuevos productos, sobre todo en depósitos y fondos.

El IPC de la Bolsa Mexicana de Valores y el Índice de Rendimiento Total (IRT) son dos indicadores principales del mercado accionario mexicano. El IPC expresa el rendimiento del mercado accionario en función de las variaciones de precio de una muestra balanceada, ponderada y representativa de acciones listadas y cotizadas en la BMV. El IRT, a su vez, incorpora al rendimiento del IPC, el pago de dividendos que realizan las emisoras que forman parte de su muestra.

Este IPC ${ }^{1}$ constituye un indicador de las fluctuaciones del mercado accionario, gracias a dos conceptos fundamentales: 1) representatividad de la muestra en cuanto a la operatividad del mercado (que es asegurada mediante la selección de las emisoras líderes, determinadas éstas a través de su nivel de bursatilidad); y 2) estructura de cálculo que contempla la dinámica del valor de capitalización del mercado (representado éste por el valor de capitalización de las emisoras que constituyen la muestra del IPC). No serán consideradas para la muestra, aquellas series que se encuentran en las siguientes situaciones: series que por alguna causa se suspendan o exista la posibilidad concreta de ser suspendidas o retiradas del mercado; o si existen dos o más series de una emisora, y el acumulado de éstas suma 14\% del total del valor del IPC, sólo permanecerán las series más representativas.

La muestra del IPC de la Bolsa Mexicana de Valores se revisa anualmente, la nueva muestra se da a conocer a mediados de enero de cada año y surte efectos a partir de febrero para los siguientes doce meses. El número de series que conforman la muestra del IPC es de 35 series accionarias, las cuales podrían variar en función de los criterios de selección antes mencionados. Dichas series corresponden a siete sectores económicos: comunicaciones y transportes, comercio, construcción, industria extractiva, servicios, transformación y varios. Cada sector se encuentra integrado por una variedad de empresas, que a su vez cada una de ellas tiene una

\footnotetext{
${ }^{1}$ Aplicado en su actual estructura desde 1978.
} 
ponderación que le otorga el valor de contribución que tendrán dentro de la muestra de acuerdo con su tamaño.

La predicción en el cambio de precios siempre ha sido un tema de gran importancia en el estudio de las finanzas. Un problema importante es el efecto que se produce en periodos concretos del calendario. Para Reinganum (1991) existen patrones de comportamiento que no son compatibles con la existencia de un mercado eficiente, como son el efecto día de la semana, el efecto enero, el efecto primera mitad del mes, el efecto apertura y cierre.

El efecto día de la semana comprende un conjunto de fenómenos que en un determinado día generan sistemáticamente rendimientos notablemente más elevados o bajos que el resto de los días de la semana. Aunque los efectos estacionales pueden aparecer en cualquier día de la semana, lunes y viernes son los días cuando, de existir, se observa con más frecuencia un efecto día de la semana. Así, el efecto lunes, es un fenómeno en virtud del cual en este día se registran, normalmente, rendimientos notablemente más bajos que durante el resto de la semana. Éste suele ir acompañado del efecto viernes, que supone la existencia de rentabilidades significativamente elevadas con respecto a las que se obtienen de lunes a jueves. Ambos efectos combinados dan lugar al denominado efecto fin de semana, tal y como se menciona en Sánchez (2006).

Las razones causantes del efecto día de la semana son múltiples. Depende del tipo de mercado, del periodo analizado, que una u otra causa tenga un mayor peso en la aparición del fenómeno estacional. Andrés y Fernández (2004) resumen las causas en tres grandes grupos:

a) Demoras en la transacción de operaciones y su liquidación

b) La actitud y costumbre de negociación de los inversionistas particulares e institucionales.

c) Razones relacionadas con la llegada de los flujos de información a los mercados.

Por lo que concierne al efecto enero, éste se refiere a que en ese mes existen mayores rentabilidades que en el resto de los meses, lo cual se da de manera muy especial entre las empresas pequeñas. Una hipótesis lógica es la de las pérdidas fiscales: los individuos venden en diciembre acciones que pueden producir minusvalías en su 
renta, presionando a la baja; en enero esta presión desaparece, produciéndose una recuperación. Este efecto puede ser más importante en las empresas pequeñas.

En cuanto al efecto primera mitad del mes, parece que es en este periodo cuando se producen las subidas, aportando muy poco la segunda mitad. Finalmente, se aprecian comportamientos diferentes según las horas del día, entre los que destaca la subida en los minutos cercanos al cierre.

Las anteriores situaciones son las que delimitan a los inversionistas y analistas financieros, al mismo tiempo proporcionan nueva información que les ayude a la toma de decisiones en lo que respecta a la formación de portafolios de inversión, así como a la compra y venta de acciones.

A lo largo del tiempo se han realizado estudios que buscan comprobar la rapidez y momento en que las acciones ajustan sus precios a los movimientos del mercado. Badrinath, Kale y Noe (1995) encontraron que los retornos mensuales de las acciones que pertenecen a instituciones de inversión se adelantan al resto; sin embargo, pruebas adicionales realizadas por McQueen, Pinegar y Thorley (1996) ponen en duda dichos resultados.

Posteriormente, Chang, McQueen y Pinegar (1999) estudiaron cinco mercados asiáticos y el de Estados Unidos, extendiendo los trabajos realizados por Lo y MacKinlay (1990a, 1990b), Mech (1993), Boudoukh, Richardson y Whitelaw (1994) y Badrinath, Kale y Noe (1995), donde se explica la habilidad que tienen los retornos pasados de un portafolios armado con empresas grandes para explicar los retornos de uno de ellos armado con empresas pequeñas, tomando en cuenta únicamente sus volúmenes y no el tipo de empresa al que pertenecen las acciones.

Chordia y Swaminathan (2000) demostraron que los retornos de las acciones con volúmenes de negociación altos se adelantan a los retornos de las acciones con volúmenes de negociación bajos, principalmente porque los volúmenes grandes de acciones se ajustan más rápidamente a la información del mercado.

Por su parte, Marshall y Walter (2002) analizaron la predictibilidad de corto plazo para portafolios de acciones negociadas en la bolsa de comercio de Santiago de Chile. El objeto de su estudio fue los retornos logarítmicos semanales para el periodo 1991-2000 de portafolios agrupados por tamaño y por volúmenes; para ello, 
utilizaron datos semanales y la muestra se examinó simultáneamente por tamaño y volumen negociado. Los retornos semanales se calcularon como diferencias en el logaritmo de los precios de los días miércoles ajustados por variaciones de capital. Para estos especialistas, el principal objetivo fue comparar la velocidad con que la información fue incorporada en los precios de acciones con diferentes características. De igual forma, encontraron que para retornos semanales existe un significativo nivel de autocorrelación y de correlación serial cruzada; además, los retornos de grandes empresas con liquidez anticipan significativamente al resto. Esta correlación serial cruzada no se debe a problemas de falta de liquidez porque es robusta en todas las particiones utilizadas.

\section{Metodología}

Los datos utilizados en este estudio corresponden a los retornos semanales de los sectores económicos de la Bolsa Mexicana de Valores de enero de 1998 a diciembre de 2007. Estos retornos se calculan como diferencia de los retornos logarítmicos semanales por sector económico (tasas instantáneas semanales), de miércoles a miércoles. Una tasa instantánea es la tasa anual convertible cada instante, es decir, aquélla que se capitaliza un infinito número de veces en un periodo. La razón de utilizar un día a mediados de la semana es para poder minimizar la probabilidad de feriados, así como para tener ausencia de transacciones por más de dos días consecutivos, como pasaría de un viernes a un lunes, tal y como se menciona en Marshall y Walker (2002). Asimismo, se utilizó el rendimiento semanal del mercado (IPC) con retraso.

La muestra se dividió por sectores económicos que se clasificaron en sectores fuertes y débiles con base en el peso de éstos en la muestra del IPC, los cuales se determinan a través de su nivel de bursatilidad y su valor de capitalización. Para la clasificación de los sectores, se tomaron las 35 acciones que representan el IPC de la Bolsa Mexicana de Valores detectando el sector económico al que pertenecían; de tal forma que el mayor peso de la muestra del IPC (10\% o más) lo conformaban los sectores fuertes (comunicaciones y transportes, comercio, construcción y extractiva), quedando los débiles (servicios, transformación y varios) menores al 10\%.

Con el propósito de analizar la velocidad de ajuste en el precio de las acciones por sector económico y, al mismo tiempo, controlar los efectos comunes, se utilizó — como se señaló anteriormente - la regresión sugerida por Marshall y Walker (2002), que a su vez retoman de Dimson (1979). La forma de esta ecuación es: 


$$
r_{B t}-r_{A t}=C+\sum_{j=-k}^{k} b_{j} r_{m t-j}+\epsilon_{t}
$$

Donde $r_{B t}-r_{A t}$ es la diferencia de los retornos logarítmicos semanales por sector económico; $B$ es el sector fuerte y $A$ el sector débil; $C$ representa una constante; $r_{m t-j}$ es el rendimiento semanal del mercado con retraso en el tiempo $t ; C_{t}$ es un error aleatorio; $b_{j} j=-k, \ldots 0, \ldots k$ son coeficientes desconocidos. La variable dependiente es la diferencia entre los sectores, y las variables independientes son el índice de mercado, ponderado y no ponderado contemporáneo con cinco periodos de rezago, cinco periodos de adelanto y en tiempo $t$.

Las variables, tanto dependientes como independientes, utilizadas en el modelo de regresión (1) se comportan como una serie de tiempo, la cual es una secuencia de los valores que asume una determinada variable o conjunto de variables, ordenadas cronológicamente y utilizadas para evaluar su comportamiento.

Según Chordia y Swaminathan (2000), el modelo de regresión (1) permite analizar la velocidad relativa de ajuste de los retornos $A$ y $B$ dado un retorno de mercado. El sector $B$ ajusta más rápidamente que el sector $A$ que es más lento en el ajuste.

Los estadísticos utilizados para evaluar los resultados obtenidos con la aplicación del modelo de regresión Dimson (1979) y Chordia y Swaminathan (2000) son la prueba $\mathrm{F}$ para el nivel de significación del modelo o contraste conjunto de nulidad de todos los parámetros, el estadístico Durbin-Watson y el coeficiente de determinación $\left(\mathrm{R}^{2}\right)$.

\section{Resultados}

Antes de realizar las regresiones, se decidió hacer un análisis de estadística descriptiva, que consta de analizar la media, desviación estándar, coeficiente de variación y las correlaciones de los sectores económicos. Lo anterior con la finalidad de presentar de forma general la relación con el IPC y detectar ex ante si existe algún hecho destacable.

De esta forma, en el cuadro 1 se muestra los estadísticos básicos semanales, de miércoles a miércoles, por sector económico, como la media, desviación estándar, coeficiente de variación y la correlación que existe entre cada sector con el IPC. 
Cuadro 1

Estadísticos básicos por sectores económicos

\begin{tabular}{ccccc}
\hline Sector & $\begin{array}{c}\text { Correlación } \\
\text { con IPC }\end{array}$ & Media & Desv.Std & Coef. Var \\
\hline IPC & & 0.0035 & 0.0352 & 10.1640 \\
EX & 0.51 & 0.0041 & 0.0512 & 12.3391 \\
CONS & 0.83 & 0.0026 & 0.0389 & 14.9994 \\
COM & 0.80 & 0.0027 & 0.0373 & 13.6050 \\
CYT & 0.93 & 0.0047 & 0.0412 & 8.7418 \\
TRA & 0.83 & 0.0016 & 0.0251 & 15.5296 \\
SER & 0.72 & 0.0043 & 0.0420 & 9.7343 \\
VAR & 0.82 & 0.0011 & 0.0365 & 34.2593 \\
\hline
\end{tabular}

Las siglas de los sectores son como sigue: industria extractiva (Ex), construcción (CONS), comercio (COM), comunicaciones y transportes (CYT), trasformación (TRA), servicios (SER) y varios (VAR). los estadísticos básicos para cada sector son: su correlación con el IPC, media, desviación estándar (Desv.Std) y coeficiente de variación (Coef. Var).

Como se observa en el cuadro 1, los sectores con mayor correlación al IPC son el de comunicaciones y transportes (CYT) con un 93\%; construcción (CONS) y transformación (TRA), con $83 \%$ cada uno; los dos primeros son del sector fuerte y el tercero del débil. El de menor correlación con el IPC es el de la industria extractiva (EX), con un $51 \%$, perteneciendo al sector fuerte.

En este estudio se midió el ajuste en el precio de las acciones agrupadas por sector económico, tal como se mencionó en la metodología, comparándolo con los retornos semanales del IPC con cinco retrasos, cinco adelantos y en tiempo $t$. Para realizar lo anterior, se aplicó el modelo de regresión (1) para cada combinación de sector fuerte con sector débil. El número de observaciones para cada regresión fue de 510 observaciones, las cuales representan los retornos semanales de enero de 1998 a diciembre de 2007 de los siete sectores que forman IPC.

En el cuadro 2 se muestran los resultados de la aplicación de la regresión (1), donde podemos destacar los valores obtenidos por el sector comunicaciones y transportes (CYT), que son muy satisfactorios en sus combinaciones con los tres sectores débiles (transformación, servicios y varios) para la comprobación de este modelo. Para el caso específico de comunicaciones y transportes (CYT) con transformación (TRA), el coeficiente de determinación es de 0.376, que para el número de grados 
de libertad es alto. Con lo anterior podemos decir que existe una respuesta por parte de los sectores a la rentabilidad del mercado, medido en este caso por el IPC. Cabe destacar que los mejores resultados para las pruebas de sector fuerte con sector débil son los obtenidos por el sector comunicaciones y transportes, obteniendo para cada bloque los valores más altos en el coeficiente de determinación, que está cercano o es mayor a lo requerido con valores satisfactorios para los demás estadísticos.

Otro de los sectores que obtuvo un resultado destacado es el de construcción (CONS) en su combinación con el sector transformación (TRA). Como se puede ver en el cuadro 2, el coeficiente de determinación $\left(\mathrm{R}^{2}\right)$ está marcado con dos asteriscos debido a que su valor es de 0.179 cumpliendo las características antes mencionadas. De igual forma, el estadístico F tiene un valor de 9.856 con un nivel de significancia cercano a cero. Sin embargo, este mismo sector, al ser comparado con los sectores servicios (SER) y varios (VAR), obtiene valores deficientes; por ejemplo, con el sector servicios (SER) el valor de $\mathrm{R}^{2}$ es de 0.024. El nivel de significancia para la prueba $\mathrm{F}$ está por encima del 0.05 que debería tener para ser significativo, por lo que lo que se rechaza el ajuste del modelo. Es importante señalar que la combinación construcción (CONS) con servicios (SER) tiene los valores más bajos para las pruebas de toda la muestra.

A lo largo de todas las pruebas el estadístico Durbin Watson se mantiene dentro de su valor óptimo (cercano a dos), lo que nos indica que no existe autocorrelación para ninguna combinación de sector fuerte con sector débil. 
Cuadro 2

Regresión Dimson

\begin{tabular}{cccccc}
\hline Sectores & & $\mathrm{R}^{2}$ & $\mathrm{DW}$ & $\mathrm{F}$ & Sign. \\
\hline EX & & 0.038 & 2.066 & 1.767 & 0.057 \\
CONS & TRA & $0.179 * *$ & $2.069^{*}$ & $9.856^{*}$ & $0.000 *$ \\
COM & & 0.145 & $2.246^{*}$ & $7.704 *$ & $0.000 *$ \\
CYT & $0.376 * *$ & $1.906 *$ & $27.281 *$ & $0.000 *$ \\
\hline EX & & 0.027 & 1.927 & 1.254 & 0.248 \\
CONS & SER & 0.024 & 2.009 & 1.09 & 0.367 \\
COM & & 0.062 & 1.911 & 2.987 & $0.001 *$ \\
CYT & & 0.109 & 1.936 & $5.544 *$ & $0.000 *$ \\
\hline EX & & 0.034 & 2.066 & 1.586 & 0.099 \\
CONS & VAR & 0.039 & 1.995 & 1.845 & 0.044 \\
COM & & 0.052 & 2.212 & 2.492 & $0.005 *$ \\
CYT & & 0.127 & $1.964 *$ & $6.578 *$ & $0.000 *$
\end{tabular}

Resultados de la ecuación de regresión (1) de miércoles a miércoles. La primera columna corresponde a los sectores fuertes y la segunda al sector débil con el que se está comparando. Juntos forman la diferencia de los retornos logarítmicos (variable dependiente). $\mathrm{R}^{2}$ es el coeficiente de determinación; DW es el estadístico Durbin Watson; F es el estadístico de la prueba de F; y la última columna es el nivel de significancia de la prueba F. Los signos $(*)$ y (**) corresponden a resultados óptimos para los estadísticos y resultados superiores a estudios anteriores, respectivamente. Todas las pruebas fueron realizadas con un nivel de significancia del $5 \%$.

Después del análisis de todos los resultados se encontró que el sector comunicaciones y transportes presenta los resultados más sobresalientes de toda la muestra; es decir, estadísticos óptimos que explican la evidencia de que este sector ajusta sus precios más rápido que los sectores transformación, servicios y varios (sectores débiles). Se desprende de aquí que los sectores fuertes ajustan más rápidamente sus precios que los sectores débiles, como lo comprobaron Chordia y Swaminathan (2000) y Marshall y Walker (2002). 


\section{Conclusiones}

Con base en los resultados obtenidos de las regresiones, observamos que el comportamiento del precio de las acciones agrupadas por sector económico de la Bolsa Mexicana de Valores manifiesta que efectivamente los retornos de los sectores fuertes se adelantan a los retornos de los sectores débiles, principalmente porque los sectores fuertes ajustan más rápidamente sus precios a los movimientos del mercado.

Este mismo resultado ocurre en los estudios realizados por Marshall y Walker (2002), así como en los de Brennan, Jegadeesh y Swaminathan (1993) y posteriormente en los de Campbell, Lo y Craig (1997), por lo que podemos concluir que el resultado de nuestro estudio es coherente con los efectuados por los autores mencionados anteriormente.

Destaca el resultado obtenido del sector comunicaciones y transportes, pues presenta el mejor coeficiente de determinación $\mathrm{R}^{2}$ en las tres regresiones realizadas con los sectores de transformación, servicios y varios. Aunado a ello, es el que siempre obtiene un nivel de significación de cero para la prueba F, por lo que podemos concluir que es el sector que ajusta más rápido sus precios con respecto a la información del mercado.

Una futura línea de investigación sería realizar este mismo estudio con los diferentes rendimientos intersemanales (lunes a lunes, martes a martes, etc.) y comparar los resultados obtenidos con la finalidad de detectar si los resultados del ajuste de miércoles a miércoles permanece constante entre los diferentes días de la semana o si existen variaciones, así como constatar los efectos día de la semana.

Nos parece importante mencionar que, con base en los resultados obtenidos, sería interesante ahondar las investigaciones sobre el sector comunicaciones y transportes y su influencia en el Índice de Precios y Cotizaciones a través del análisis de la velocidad de ajuste entre portafolios-sectoriales según la clasificación utilizada durante nuestro estudio. 


\section{Referencias}

Anderson, D., D. Sweeney y T. Williams (2005). Estadística para administración y economía. $8^{\mathrm{a}}$ ed., México: Thomson.

Andrés, J. y A. Fernández (2004). "Análisis de la estacionalidad diaria en el Mercado español de bonos y obligaciones del Estado". Boletín de Información Comercial Española, $\mathrm{N}^{\mathrm{o}} 2804$, pp. 21-29.

Badrinath, S. G., J. R. Kale y T. H. Noe (1995). "Of Shepherds, Sheep, and the Cross-autocorrelations in Equity Returns". Review of Financial Studies, Vol. 8, No 2, pp. 401-430.

Berenson, M., D. Levine y T. Krehbiel (2006). Estadística para administración. $4^{\mathrm{a}}$. ed., México: Prentice Hall.

Bolsa Mexicana de Valores (2008). Indices sectoriales de la Bolsa Mexicana de Valores. Recuperado en enero 20 de 2008:

http://www.bmv.com.mx/

Boudoukh, J., M. P. Richardson y R. F. Whitelaw (1994). "A Tale of Three Schools: Insights on Autocorrelations of Short-Horizon Returns". Review of Financial Studies, Fall, Vol. 7, № 3, pp. 539-573.

Brealey R. y S. Myers (1995). Principios de finanzas corporativas. $5^{\text {a }}$ ed., Madrid: McGraw Hill.

Brennan, M. J., N. Jegadeesh y B. Swaminathan (1993). "Investment Analysis and the Adjustment of Stock Prices to Common Information". Review of Financial Studies, Vol. 6, № 4, pp. 799-824.

Campbell, J.Y., A. W. Lo y M. K. Craig (1997). The Econometrics of Financial Markets. Princeton, New Jersey: Princeton University Press.

Chang E., G. McQueen y J. Pinegar (1999). "Cross-autocorrelation in Asian Stock Markets”. Pacific-Basin Finance Journal, Vol. 7, No 5, pp. 471-494. 
Chordia, T. y B. Swaminathan (2000). "Trading Volume and Cross-Autocorrelations in Stock Returns". Journal of Finance, Vol. 55, No 2, pp. 913-935.

Dimson, E. (1979). "Risk Measurement when Shares are Subject to Infrequent Trading". Journal of Financial Economics, Vol. 7, pp. 197-226.

FAmA, E. (1970). "Efficient Capital Markets: A Review of Theory and Empirical Work". Journal of Finance, Vol. 25, N 2, pp. 383-417.

Gómez, F. (2000). Gestión de carteras (eficiencia, teoría de cartera, CAPM, APT). $2^{\mathrm{a}}$ ed., España: Desclée de Brouwer.

Gujarati, D. (1997). Econometría. $3^{\text {a }}$ ed., Bogotá, Colombia: McGraw Hill.

Kendall, M. G. (1953). "The Analysis of Economic Time Series, Part I: Prices". Journal of the Royal Statistical Society, Vol. 116, pp. 11-34.

Lo, A. y A. MacKinlay (1990a). "An econometric analysis of nonsynchronous trading”. Journal of Econometrics, Vol. 45, № 1, pp.181-211.

(1990b). "When are Contrarian Profits Due to Market Overreaction?" Review of Financial Studies, Vol. 3, No 2, pp. 175-205.

López, R. (2002). Comprendiendo las matemáticas financieras. Puebla, México: Universidad de las Américas.

Mandelbrot, B. (1963). "The Variation of Certain Speculative Prices”. Journal of Business, October, Vol. 36, pp. 394-419.

Marshall, P. y E. Walker (2001). "Asymmetric Reaction to new Information and Serial Dependence of Short-Run Returns". Journal of Applied Economics, Vol. 5, No 2, pp. 273-292.

(2002). "Volumen, tamaño y ajuste a nueva información en el mercado accionario chileno". Estudios de Economía, Vol. 29, № 2, pp. 247-268.

MARTínez, E. (2000). Invertir en bolsa (conceptos y estrategias). España: McGraw Hill Interamericana. 
McQueen, G., M. Pinegar y S. Thorley (1996). "Delayed Reaction to Good News and the Cross-Autocorrelation of Portfolio Returns". Journal of Finance, Vol. 51, N 3, pp. 889-919.

Месн, T. S. (1993). "Portfolio Return Autocorrelation". Journal of Financial Economics, Vol. 34, pp. 307-344.

Osborne, M. F. M. (1959). "Brownian Motion in the Stock Market". Operations Research, Vol. 7, No 2, pp. 145-173.

Pulido, A. y A. López (1999). Predicción y simulación aplicada a la economía y gestión de empresas. Madrid, España: Pirámide.

Reinganum, M. R. (1991). "El colapso de la hipótesis del mercado eficiente". Análisis Financiero, Vol. 55, pp. 30-37.

Roberts, H. (1959). "Stock Market "Patterns" and Financial: Analysis. Methodological Suggestions". Journal of Finance, Vol. 14, No 1, pp. 1-10.

Rodríguez, L. M. y J. S. Fermín (2006). Mercado eficiente y caminata aleatoria en la Bolsa de Valores de Caracas. INCI, Vol. 31, N 12, pp. 888-893.

Ross, S., R. Westerfield y J. JafFe (1995). Finanzas corporativas. $3^{\text {a }}$ ed., México: Irwin.

SÁnchez, J. (2006). "Los efectos día de la semana, mes del año y cambio de año en los rendimientos diarios de bonos del Estado a medio y largo plazo de la euro zona". Boletín de Información Comercial Española, No 2891, pp. $31-44$.

VIDAURRI, H. (2004). Matemáticas financieras. $3^{\text {a }}$ ed., México: Thomson. 